\title{
Hidrocele secundario a pancreatitis necrohemorrágica: a propósito de un caso
}

\author{
Hydrocele secondary to necrohemorrhagic pancreatitis: a case report \\ Hidrocele secundária à pancreatite necrohaemorrágica: um relato de caso
}

\author{
Fernando Silva Michalón (1) ', Danny Fernando Silva Cevallos $\mathbb{D}^{2}{ }^{2}$, Alexia Peréz Vivar $\mathbb{( D )}$ ', Ruddy \\ Solange García Rosero (1D) 1
}

\begin{abstract}
1 Médico General, Universidad Católica de Santiago de Guayaquil, Guayaquil-Ecuador; ${ }^{2}$ Servicio de Medicina Interna, Emergencias y Hospitalización, Clínica Guayaquil, Guayaquil - Ecuador.
\end{abstract}

\section{Correspondencia a:}

Fernando Silva Michalón, fsilvamichalon@gmail.com

Recibido: 30 de septiembre, 2021 Aceptado: 7 de octubre, 2021 Publicado: 18 de noviembre, 2021

\section{CASO CLÍNICO}

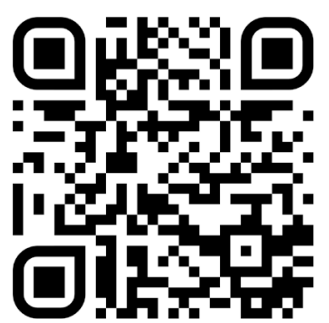

Escanea en tu dispositivo móvil o revisa este artículo en: https:// revistaclinicaguayaquil.org

\section{RESUMEN}

La pancreatitis aguda es una enfermedad cuyo estadio inicial consiste en una reacción inflamatoria, de los cuales entre el 30\% y $50 \%$ de los casos evoluciona a disfunción pancreática severa y/o falla multiorgánica. Además, pueden desarrollar complicaciones pancreáticas o extrapancreáticas, dentro las cuales se encuentra el hidrocele de origen pancreático, una complicación a distancia y poco frecuente. Reportamos un caso de pancreatitis necrohemorrágica en un paciente masculino de 48 años, con antecedente de pancreatitis de origen biliar hace 4 años, con dolor abdominal acompañado de edema testicular. El presente reporte describe una complicación a distancia de la pancreatitis aguda, su baja incidencia y escasos reportes en la literatura justifican la presentación de este caso.

Palabras clave: Pancreatitis; Pancreatitis aguda; Hidrocele testicular; Necrosis pancreática

\begin{abstract}
Acute pancreatitis is a disease in which its initial stage consists of an inflammatory reaction; between $30 \%$ and $50 \%$ of the cases progress to severe pancreatic dysfunction and/or multi-organ failure. In addition, it can develop pancreatic or extrapancreatic complications, among which is the hydrocele of pancreatic origin, a rare and distant complication. We report a case of necrohemorrhagic pancreatitis in a 48-year-old male, with a history of biliary pancreatitis 4 years before, with abdominal pain accompanied by testicular edema. This report describes a distant complication of acute pancreatitis, its low incidence and few reports in the literature justify the presentation of this case.
\end{abstract}

Key words: Pancreatitis; Acute pancreatitis; Testicular hydrocele; Pancreatic necrosis

\section{RESUMO}

A pancreatite aguda é uma doença cujo estágio inicial consiste em uma reação inflamatória, da qual $30 \%$ a $50 \%$ dos casos progridem para disfunção pancreática grave e/ou falha de múltiplos órgãos. Além disso, podem se desenvolver complicações pancreáticas ou extrapancreáticas, incluindo a hidrocele de origem pancreática, uma rara complicação distante. Relatamos um caso de pancreatite necrohaemorrágica em um paciente masculino de 48 anos com história de pancreatite de origem biliar há 4 anos, com dor abdominal acompanhada de edema testicular. O presente relatório descreve 
uma complicação distante da pancreatite aguda, sua baixa incidência e relatórios escassos na literatura justificam a apresentação deste caso.

Palavras-chave: Pancreatite; Pancreatite aguda; Hidrocele testicular; Necrose pancreática

\section{INTRODUCCIÓN}

La pancreatitis aguda es un proceso inflamatorio de causas múltiples, asociado principalmente a antecedentes de litiasis de vesícula biliar y vías biliares, así como al consumo de alcohol (1). La pancreatitis aguda se produce por la activación precoz de enzimas pancreáticas, principalmente la tripsina, liberada en las células acinares. De acuerdo a la clasificación de Atlanta del año 2012 se clasifica en leve, moderadamente grave y grave; siendo un $80 \%$ de los casos de carácter leve. El porcentaje restante corresponde a la presentación clínica grave, presentando un $50 \%$ de mortalidad (1). Para su diagnóstico, se consideran 2 de 3 criterios, entre los cuales se encuentran: dolor abdominal en epigastrio irradiado a la espalda, valores de amilasa y lipasa hasta 3 veces elevados de su rango normal y/o hallazgos en imágenes de abdomen (2). Quienes superan la fase crítica de la enfermedad pueden presentar, además de complicaciones locales, complicaciones sistémicas y falla multiorgánica (3). Sin embargo, la presentación concomitante de un hidrocele de origen pancreático es una entidad con escasos reportes en la literatura. El objetivo de este reporte es dar a conocer el caso de un paciente con hidrocele secundario a pancreatitis necrohemorrágica.

\section{DESCRIPCIÓN DEL CASO CLÍNICO}

Paciente masculino de 48 años con antecedente de litiasis biliar y pancreatitis aguda hace 4 años, que acude a emergencia por presentar, luego de la ingesta de comida copiosa, dolor abdominal agudo, tipo cólico de intensidad variable, sin mejoría pese a la administración de analgésicos vía oral y que, 24 horas más tarde, presenta edema testicular unilateral.

Al examen físico se encuentra despierto y orientado, con las mucosas semihúmedas y subictéricas. Abdomen globuloso, distendido, levemente blando y poco depresible, doloroso a la palpación profunda, además de presentar signo de rebote y Blumberg positivos. Extremidades simétricas, no edematosas. Se observa edema testicular (figura 1), y se palpa masa no reducible en el testículo izquierdo.
Presenta un quick SOFA score (quick Sepsis related Organ Failure Assessment, qSOFA) de 2.

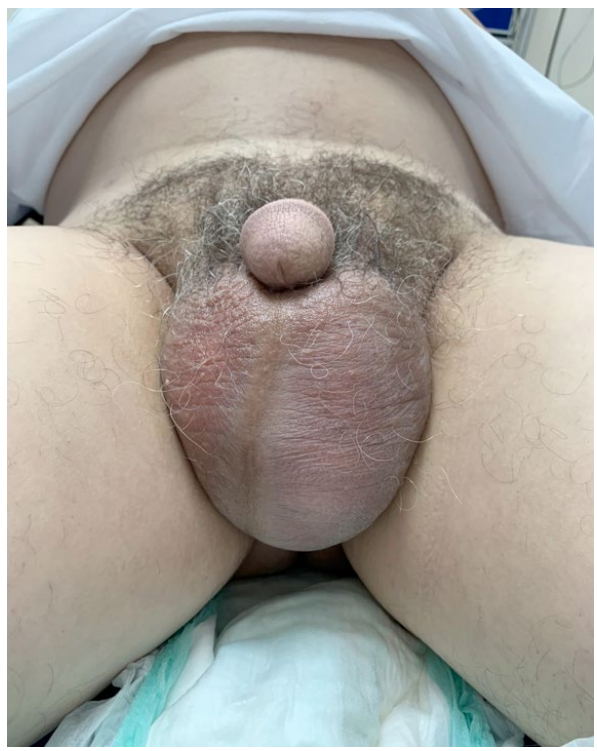

Figura 1. Edema testicular izquierdo.

Se solicitan estudios de laboratorio (tabla 1) e imágenes. La tomografía axial computarizada (TC) de tórax muestra derrame pleural bilateral, a predominio izquierdo, y lesiones basales bilaterales (figuras 2 A y B). En la TC de abdomen y pelvis se observa el páncreas aumentado de tamaño, heterogéneo, con presencia de una colección intra pancreática, necrosis peri pancreática en gran cantidad y ligera cantidad de líquido libre en cavidad abdominal (figura 3). Además, se logra visualizar una masa en testículo izquierdo (figura 4). La ecografía testicular describe un hidrocele y epididimitis izquierda.

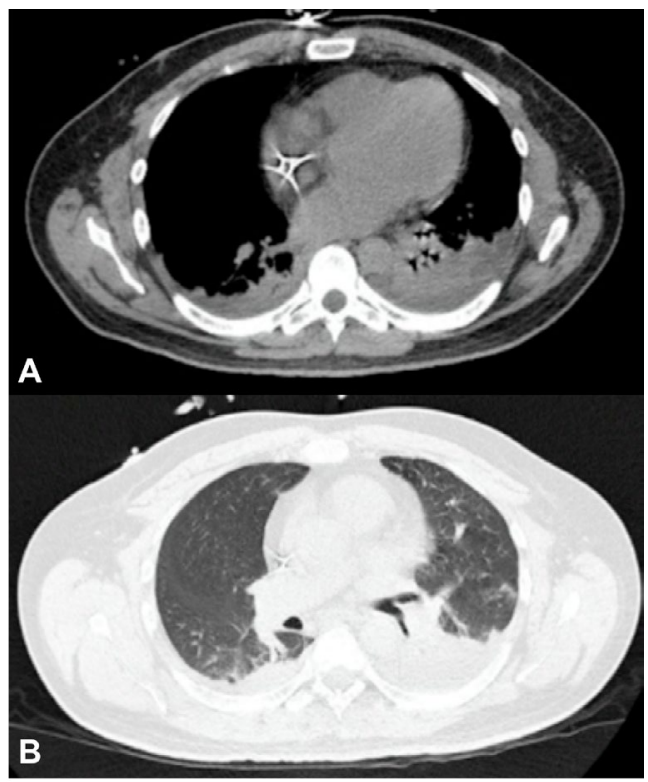

Figura 2. Imagen de tomografía computarizada (A) ventana mediastínica; (B) ventana pulmonar. Imágenes hiperdensas bibasales correlacionadas con derrame pleural bilateral, a predominio del lado izquierdo. Broncograma aéreo, presencia de infiltrados pulmonares por proceso neumónico. 


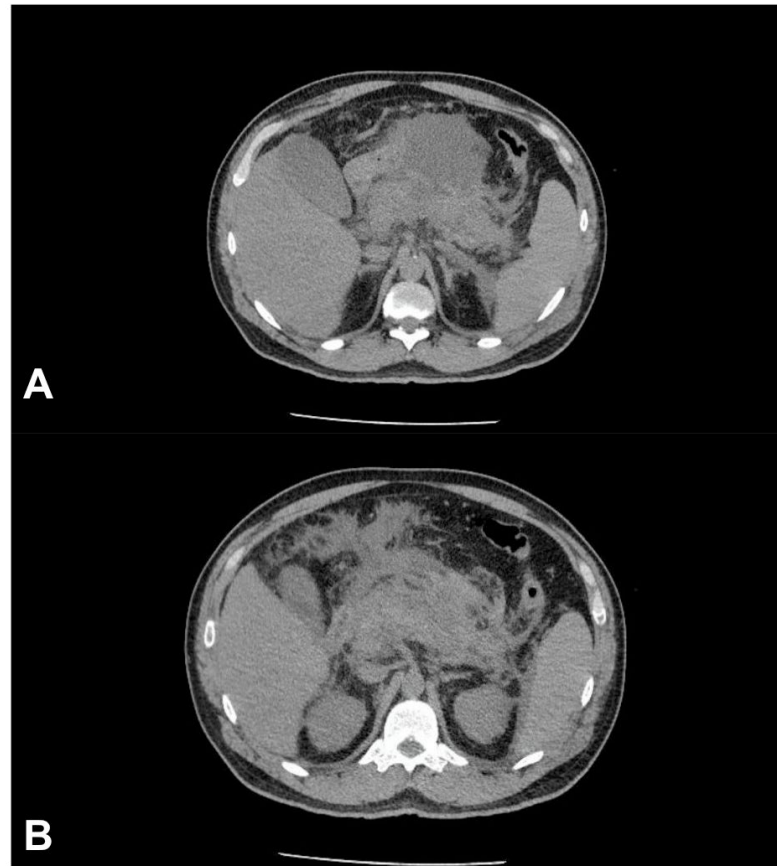

Figura 3. Imagen de tomografía computarizada (A) ventana mediastínica L2; (B) ventana mediastínica L3. Se observa edema con exudado peripancreático severo, aumento difuso de la glándula, colección pancreática definida no encapsulada, destrucción del parénquima pancreático y leve colección de gas retroperitoneal.

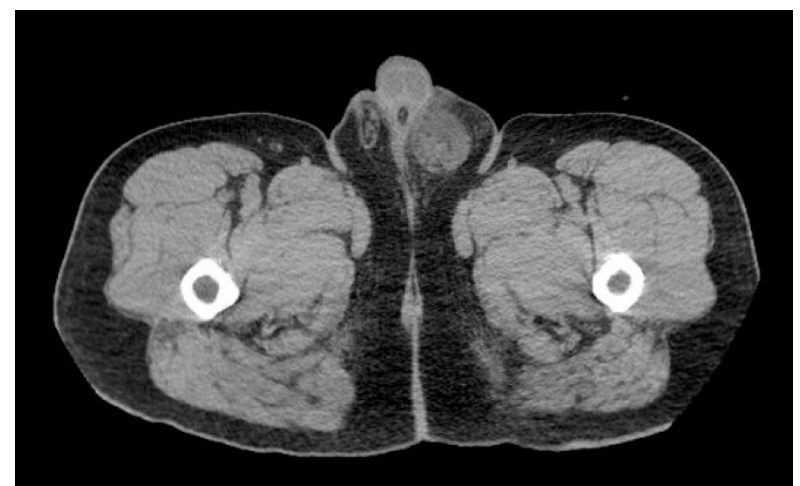

Figura 4. Imagen de tomografía computarizada, se evidencia infiltración de escroto izquierdo.

Tabla 1: Exámenes de laboratorio.

\begin{tabular}{|c|c|c|c|c|c|}
\hline Laboratorio & $\begin{array}{l}\text { Valor de } \\
\text { referencia }\end{array}$ & Día 1 & Día 2 & Día 3 & Día 4 \\
\hline Leucocitos, x 109/l & $4.5-10$ & 10.8 & 23.6 & 21.3 & 61.3 \\
\hline Hemoglobina, g/dl & $14-18$ & 13.0 & 12.0 & 10.7 & 11.6 \\
\hline Hematocrito, \% & $42-52$ & 39.2 & 38.0 & 31.0 & 35.9 \\
\hline Neutrófilos, x 109/I & $2.2-4.8$ & 9.7 & 20.1 & 18.3 & 57.9 \\
\hline Plaquetas, $\mathrm{mm}^{3}$ & $\begin{array}{c}130.000- \\
400.000\end{array}$ & 99.000 & 74.000 & 83.000 & 115.000 \\
\hline $\begin{array}{c}\text { Tiempo protrombina, } \\
\text { segundos }\end{array}$ & $10.8-14.5$ & 14.1 & 23.0 & 23.0 & 12.9 \\
\hline $\begin{array}{l}\text { Tiempo parcial de } \\
\text { tromboplastina, } \\
\text { segundos }\end{array}$ & $25-35$ & 21.0 & 21.0 & 21.0 & 20.1 \\
\hline $\begin{array}{c}\text { International } \\
\text { normalized ratio (INR) }\end{array}$ & $0.9-1.3$ & 1.18 & 1.95 & 1.95 & 1.07 \\
\hline Potasio, meq/l & $3.5-5.1$ & 3.6 & 4.8 & 5.2 & 4.0 \\
\hline Sodio, meq/l & $135-148$ & 135.0 & 139.0 & 145.5 & 142.7 \\
\hline Fósforo, mg/dl & $2.7-4.5$ & 1.9 & 4.9 & 10.9 & 2.8 \\
\hline Calcio, mg/dl & $8.6-10$ & 6.8 & 8.9 & 7.9 & 8.0 \\
\hline Magnesio, mg/dl & $1.6-2.6$ & 1.6 & 1.5 & 2.3 & 1.6 \\
\hline Glucosa, mg/dl & $70-100$ & 124 & 128 & 178 & 253 \\
\hline Urea, mg/dl & $12.6-42.6$ & 68 & 63 & 94 & 91 \\
\hline Creatinina, mg/dl & $0.4-1.2$ & 1.8 & 3.2 & 3.6 & 1.3 \\
\hline
\end{tabular}




\begin{tabular}{|c|c|c|c|c|c|}
\hline Laboratorio & $\begin{array}{l}\text { Valor de } \\
\text { referencia }\end{array}$ & Día 1 & Día 2 & Día 3 & Día 4 \\
\hline $\begin{array}{c}\text { Aspartato } \\
\text { transaminasa, } \mathrm{u} / \mathrm{l}\end{array}$ & $15-41$ & 33 & 10 & 10 & 409 \\
\hline $\begin{array}{c}\text { Alanina } \\
\text { transaminasa, } \mathrm{u} / \mathrm{l}\end{array}$ & $10-40$ & 15 & 1592 & 1592 & 1120 \\
\hline $\begin{array}{c}\text { Fosfatasa alkalina, } \\
\qquad \mathrm{kat} / \mathrm{l}\end{array}$ & $42-128$ & 66 & 76 & 128 & 192 \\
\hline $\begin{array}{l}\text { Bilirrubina total, mg/ } \\
\mathrm{dl}\end{array}$ & $0-1.1$ & 0.6 & 2.1 & 2.5 & 3.6 \\
\hline $\begin{array}{c}\text { Bilirrubina directa, } \\
\text { mg/dl }\end{array}$ & $0.1-0.5$ & 1.6 & 1.9 & 1.9 & 3.2 \\
\hline $\begin{array}{c}\text { Bilirrubina indirecta, } \\
\mathrm{mg} / \mathrm{dl}\end{array}$ & $0.2-0.8$ & 0.4 & 0.5 & 0.5 & 0.4 \\
\hline Amilasa, u/l & $27-131$ & 174 & 377 & 377 & 392 \\
\hline Lipasa, u/l & $22-51$ & 182 & 69 & 69 & 70 \\
\hline $\begin{array}{c}\text { Lactato } \\
\text { deshidrogenasa, UI// }\end{array}$ & $135-225$ & 786 & 777 & $N / R$ & $N / R$ \\
\hline Procalcitonina, ng/ml & $0-0.5$ & 2 & 2 & 7 & 8 \\
\hline $\begin{array}{c}\text { Proteínas totales, g/ } \\
\text { dl }\end{array}$ & $6.6-8.7$ & 5.4 & $N / R$ & 4.8 & $N / R$ \\
\hline Albúmina, g/dl & $3.5-5$ & 3.0 & 2.8 & 2.6 & 2.8 \\
\hline $\mathrm{pH}$ & $7.35-7.45$ & 7.42 & 7.36 & 7.17 & 7.42 \\
\hline $\mathrm{pCO} 2, \mathrm{mmHg}$ & $35-40$ & 31.5 & 37.7 & 29.1 & 50.7 \\
\hline $\mathrm{HCO} 3, \mathrm{mmol} / \mathrm{l}$ & $21-27$ & 20.0 & 21.1 & 10.6 & 32.3 \\
\hline $\begin{array}{l}\text { Exceso de bases, } \\
\mathrm{meq} / \mathrm{l}\end{array}$ & $-2.3-+2.3$ & -3.4 & -3.8 & -16.0 & 7.1 \\
\hline
\end{tabular}

dl: decilitros, g: gramos, HCO3: bicarbonato, I: litros, meq: miliequivalentes, mg: miligramos, ml: mililitros, mm3: milímetro cúbico, mmHg: milímetros de mercurio, mmol: milimol, N/R: no reportado, pCO2: presión parcial de dióxido de carbono en la sangre arterial, $\mathrm{pH}$ : potencial de hidrogeniones, u: microgramos, Ul: unidades internacionales, $\mu \mathrm{kat}$ : microkatal.

Sediagnosticaconpancreatitisnecrohemorrágica severa, con criterio quirúrgico. Inicia tratamiento con metronidazol, gluconato de calcio, tramadol y metoclopramida. En el momento del ingreso presentó los siguientes scores: índice clínico de gravedad en pancreatitis aguda BISAP (Bedside index for severity in acute pancreatitis) de 4 , con una mortalidad del 7.4\%; POP score (Pancreatitis Outcome Prediction) de 16, con una mortalidad del 28\%; puntuación simplificada de fisiología aguda SAPS-II (Simplified Acute Physiologic Score) de 57; SOFA de 8, fallo multiorgánico; escala de Ranson 1 (mortalidad 1.5\%); y APACHE II (Acute Physiology And Chronic Health Evaluation II) de 4, con una mortalidad de $5.1 \%$.

A las 48 horas del ingreso presenta score SOFA de 8. Se realiza medición de presión intra abdominal que revela $35 \mathrm{cmH}_{2} \mathrm{O}$, compatible con síndrome compartimental abdominal. Además, presenta leucocitosis marcada y elevación de azoados y procalcitonina, lo cual sugiere el desarrollo de necrosis infectada. Se rota terapia antimicrobiana a meropenem.

El paciente ingresa a quirófano para laparotomía exploratoria con hallazgo de necrosis pancreática y se realizó drenaje del absceso ( $1.000 \mathrm{ml}$ de pus) (figura 5). A las 72 horas de su ingreso, el paciente presenta SOFA de 16 , shock séptico refractario con acidosis metabólica, hiperlactacidemia y falla multiorgánica, que requiere soporte vasopresor con norepinefrina a $0.45 \mathrm{mg} / \mathrm{kg} / \mathrm{min}$. 


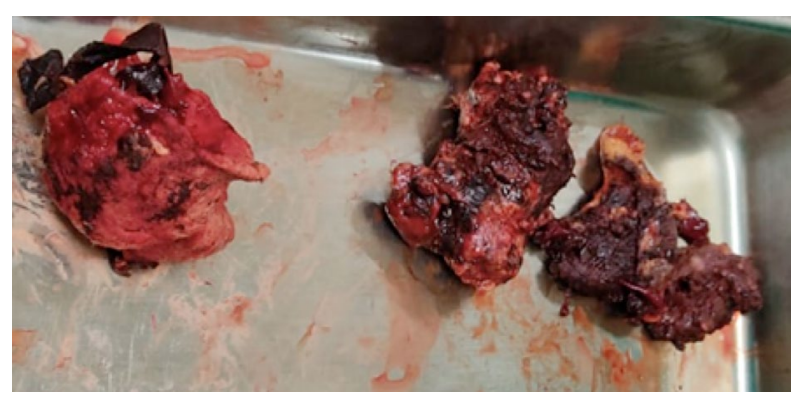

Figura 5. Se observan zonas de necrosis y hemorragia en el parénquima pancreático.

Al cuarto día, evoluciona con elevación de leucocitos de $34.000 \mathrm{ul} / \mathrm{ml}$ (reacción leucemoide). Se progresa el esquema antimicrobiano con meropenem, fluconazol, metronidazol y vancomicina, por sospecha de neumonía asociada a ventilador. Además, en la TC de abdomen se observan imágenes hiperdensas en páncreas, con sospecha de calcificaciones (figura 6). El paciente continúa en condiciones críticas con falla multiorgánica y pronóstico reservado.

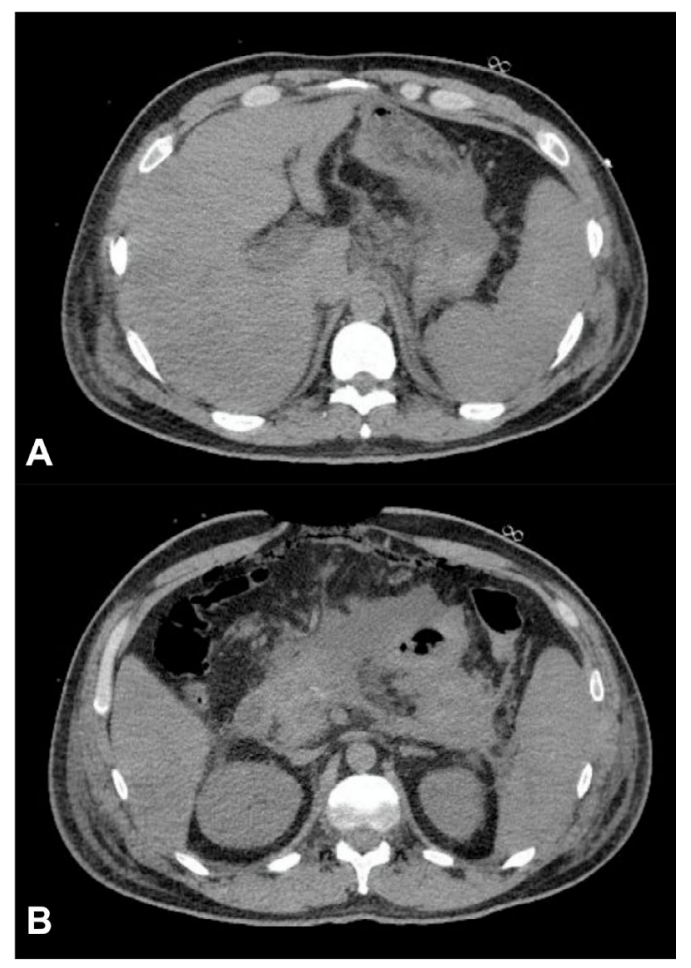

Figura 6 A y B. Imágenes de tomografía computarizada de abdomen al sexto día de ingreso donde se observa páncreas difuso con calcificaciones en su interior.

\section{DISCUSIÓN}

La pancreatitis aguda como entidad clínica debe ser sospechada en pacientes con dolor abdominal severo. Usualmente, es descrita como dolor en epigastrio que se irradia hacia el lado izquierdo en hemicinturón de Katsch o dolor en hipocondrio izquierdo. En casos severos, como el paciente descrito, se presenta como un abdomen agudo; además, el paciente ingresó con taquipnea, taquicardia y $\mathrm{SpO} 2$ de
94\% lo que hace sospechar de un cuadro severo con posible fallo multiorgánico.

Las complicaciones poco frecuentes de la pancreatitis severa pueden cursar en zonas distales, en este caso fue a nivel genital, en escroto; esta puede asemejarse a una torsión testicular, epididimitis, o tumor testicular $(3,4)$. La conexión probable entre ambas patologías se explica por la continuidad anatómica del saco escrotal con el tejido pélvico extraperitoneal que conecta con el espacio pararrenal anterior izquierdo (3). De acuerdo con lo descrito por Zimin y colaboradores, en la pancreatitis aguda grave, el líquido pancreático puede transitar en el retroperitoneo a lo largo del músculo psoas hasta el escroto a través del canal inguinal (5). Fisiopatológicamente se puede explicar por el efecto inflamatorio y destructivo del líquido pancreático que genera necrosis grasa del tejido blando del escroto (6).

Al ingreso, los laboratorios no mostraron leucocitosis, pero sí marcadores de inflamación inespecífica elevados (ferritina, LDH). La amilasa se encontraba levemente elevada $y$, generalmente se encuentran valores 3 veces de su valor normal, esto se puede explicar por el tiempo de evolución del cuadro clínico (48 horas). La amilasa se eleva de 6 a 12 horas del comienzo del cuadro y tiene una vida corta de 10 horas aproximadamente, razón por la cual los niveles en el paciente se encontraban normales. La lipasa, por el contrario, regresa a sus niveles normales luego de 8 a 14 días del comienzo de los síntomas y es más sensible y específica que la amilasa, aunque también se han reportado causas extra pancreáticas de elevación de esta enzima (7).

El $22 \%$ de los casos las pancreatitis pueden recurrir, como en este caso (8). El paciente refiere un cuadro de pancreatitis aguda hace 4 años sin secuela clínica. El 15\% de las pancreatitis son necrotizantes y tienen un peor pronóstico que las pancreatitis edematosas, con $17 \%$ de mortalidad (9). La morbi-mortalidad de la evolución de una pancreatitis severa es alta, junto con este proceso inflamatorio, la proteína $C$ reactiva elevada presume una alta complejidad de cuidados intensivos y una descompensación rápida y de gravedad del paciente que conduce a una mortalidad elevada (10).

La pancreatitis grave puede evolucionar hacia una falla orgánica múltiple con compromiso sistémico. Esto está asociado con los niveles enzimáticos y el aumento de citocinas que 
actúan entre los tejidos adyacentes provocando un síndrome de respuesta inflamatoria sistémica (SIRS) con complicaciones como ascitis, derrame pleural e hidrocele $(2,3)$.

Se debe diferenciar el SIRS de una falla multiorgánica. El primero se debe a la activación de la cascada de citoquinas y si persiste, conlleva un riesgo más alto de fallo multiorgánico. Al ingreso, el paciente cumplía dos criterios de SIRS, pero al segundo día del ingreso los leucocitos mostraron elevación notable, indicando una posible infección. Desde el primer día de hospitalización, la falla renal era evidente, con elevación de nitrógeno ureico y creatinina, la cual durante los días siguientes empeoró. Al segundo día la función hepática se encontraba alterada (tabla 1), sumándose a la falla de órganos. Posteriormente, luego de necesitar vasopresores, se evidencia la elevación de proBNP, además de una procalcitonina de 7 . El paciente no respondía a vasopresores a dosis titulables, ni a la antibioticoterapia, y finalmente, realiza una reacción leucemoide.

El manejo del paciente no siguió estrictamente las guías. No se inició el tratamiento agresivamente con fluidos, pero si se analizó el ingreso a la Unidad de Cuidados Intensivos luego de valorar el cuadro clínico. Se introdujo una sonda nasogástrica para nutrición enteral y sonda vesical para cuantificar diuresis al momento del ingreso, lo que recomiendan las guías americanas de manejo de pancreatitis aguda $(9,11)$. La prescripción de antibióticos como profilaxis se realiza únicamente en casos de necrosis pancreática infectada, no está indicada en pancreatitis edematosa ni en pancreatitis necrotizante estéril $(9,10)$. Las guías indican la aspiración por aguja fina del tejido pancreático para evidenciar si existe una necrosis infectada o no debido a que el manejo de ambas entidades es diferente. A pesar de que en este paciente se realizó una necrosectomía, debido a la no mejoría del cuadro, las guías indican dicho procedimiento únicamente en casos de infección, ya que la necrosectomía en pancreatitis necrotizante aséptica aumenta la morbimortalidad (9).

Encontrar un hidrocele en una pancreatitis sugiere un desenlace de carácter reservado, en el cual la inflamación y la necrosis grasa agravan el cuadro. Hasta el momento existen 34 casos reportados en la literatura (12). En ellos se describen distintas entidades y diagnósticos diferenciales como por ejemplo, infarto testicular, torsión testicular, epididimitis, tumores testiculares, y hernia inguinal (12). Es de suma importancia descartar otras emergencias testiculares, ya que el hidrocele asociado a pancreatitis podría tener una evolución más tórpida y de peor pronóstico, mientras que las otras patologías testiculares requieren intervención quirúrgica temprana.

Las complicaciones locorregionales y distales de una pancreatitis aguda pueden estar relacionados con la gravedad del cuadro, sin embargo, la presentación de un cuadro de pancreatitis hemorrágica acompañado de hidrocele es aproximadamente menor al $1 \%$ (13). El difícil manejo y variabilidad de la evolución del paciente dicta preparar este caso para la toma de decisiones a futuro, lograr un diagnóstico precoz, oportuno y disminuir la morbimortalidad de esta patología.

\section{REFERENCIAS BIBLIOGRÁFICAS}

1. Banks PA, Bollen TL, Dervenis C, Gooszen HG, Johnson CD, Sarr MG, et al. Classification of acute pancreatitis--2012: revision of the Atlanta classification and definitions by international consensus. Gut. enero de 2013;62(1):102-11. DOI: 10.1136/gutjnl-2012-302779

2. Bustamante Durón D, García Laínez A, Umanzor García W, Leiva Rubio L, Barrientos Rosales A, Diek Romero L. Pancreatitis Aguda: Evidencia Actual. Arch Med. 2018;14(1:4):1-10. DOI: 10.1136/ gutjnl-2012-302779

3. Monterrosas-Minutti CA, Becerra-Cárdenas J, Solis-Escobedo JC, Guerrero-Nuño J, Manzo-Pérez BO, Varela-Figueroa DA, et al. Fístula escrotocutánea como complicación de pancreatitis necrosante aguda. Rev Mex Urol. 2015;75(3):16771. DOI: 10.1016/j.uromx.2015.04.002

4. Malayala SV, Sa'ati A, Raza A. Pancreatic Hydrocele: A Case Report and Review of Literature. Pancreas. octubre de 2015;44(7):1164-5. DOI: 10.1097/ MPA.0000000000000398

5. Zimin AF, Satsukevich VN, Molchanov NP. [Acute pancreatitis with hemorrhagic flow into the scrotum]. Vestn Khir Im I I Grek. marzo de 1979;122(3):47-8.

6. Kim S-B, Je B-K, Lee SH, Cha SH. Scrotal swelling caused by acute necrotizing pancreatitis: CT diagnosis. Abdom Imaging. abril de 2011;36(2):218-21. DOI:10.1007/s00261-0109638-0

7. Yadav D, Agarwal N, Pitchumoni CS. A critical evaluation of laboratory tests in acute pancreatitis. Am J Gastroenterol. junio de 2002;97(6):1309-18. DOI: 10.1111/j.1572-0241.2002.05766.x

8. Sankaran SJ, Xiao AY, Wu LM, Windsor JA, Forsmark $C E$, Petrov MS. Frequency of progression from acute to chronic pancreatitis and risk factors: a meta-analysis. Gastroenterology. noviembre 
de 2015;149(6):1490-1500.e1. DOI: 10.1053/j. gastro.2015.07.066

9. Banks PA, Freeman ML. Practice guidelines in acute pancreatitis. Am J Gastroenterol. octubre de 2006;101(10):2379-400. DOI: 10.1111/j.15720241.2006.00856.x

10. Greenberg JA, Hsu J, Bawazeer M, Marshall J, Friedrich JO, Nathens A, et al. Clinical practice guideline: Management of acute pancreatitis. Can J Surg. 2016;59(2):128-40. DOI: 10.1503/ cjs.015015

11. Tenner S, Baillie J, DeWitt J, Vege SS. American College of Gastroenterology guideline: management of acute pancreatitis. Am J Gastroenterol. septiembre de 2013;108(9):140015; 1416. DOI: 10.1038/ajg.2013.218

12. Chang $Y-H$, Weng S-Y, Shiue S-J, Cheng C-L, Wu $M-S$. Hydrocele in recurrent acute pancreatitis caused by testicular venous obstruction. Medicine (Baltimore). 2020;99(18):e19738. DOI: 10.1097/ md.0000000000019738

13. Atiq M, Budhani I, Snyder R, Safdar K, Khan A, Chauhan S. Pancreatic hydrocele: an unusual manifestation of severe acute pancreatitis. Gastrointest Endosc. agosto de 2008;68(2):393-5. DOI: 10.1016/j.gie.2007.11.030

\section{ACERCA DE LOS AUTORES}

1. Fernando Silva Michalón. Médico General, Universidad Católica de Santiago de Guayaquil, Guayaquil-Ecuador.

ORCID: 0000-0001-9603-4958

2. Danny Fernando Silva Cevallos. Servicio de Medicina Interna, Emergencias y Hospitalización, Clínica Guayaquil, Guayaquil - Ecuador.

ORCID: 0000-0003-1014-7607

3. Alexia Peréz Vivar. Médico General, Universidad Católica de Santiago de Guayaquil, Guayaquil-Ecuador.

ORCID: 0000-0002-3028-8020

4. Ruddy Solange García Rosero. Médico General, Universidad Católica de Santiago de Guayaquil, Guayaquil-Ecuador.

ORCID: 0000-0002-8539-359X 